\title{
Health Risks of Selected Mass Gatherings in the Philippines in 2015 and Implications for Public Health Preparedness
}

Ronald P. Law

Health Emergency Management Bureau, Department of Health

\begin{abstract}
Background. Mass gatherings (MG) are events that draw together a large number of people in one or several occasions happening in single or multiple places for a definite period of time. These can lead to different public health risks through exposure to infectious diseases, trauma, and environmental factors. The Philippine Department of Health (DOH) in 2015 participated in special planned events that constituted mass gatherings namely the AsiaPacific Economic Cooperation (APEC) meetings, the Black Nazarene procession, and the Papal Visit.
\end{abstract}

Objective. The study aimed to describe the different health risks arising from the three (3) identified mass gathering events in the Philippines in 2015 and relate them to public health preparedness.

Methods. This was a descriptive study of the health risks arising from the MG events. Sources of data were reports submitted by deployed medical teams to the Operations Center (Opcen) that closely monitored the MG.

Results. The study found infectious causes, trauma, temperature-related conditions, and noncommunicable diseases to be the important categories of health risks in the specified mass gatherings. These validated the common health risk categories observed in previously well-studied mass gatherings.

Conclusion. The study highlighted important health risks and factors for consideration in public health preparedness for mass gatherings in terms of appropriate and effective public health strategies that should be established to minimize health risks and reduce health system impacts of mass gatherings.

Key Words: disasters, disaster medicine, DRRM (disaster risk reduction and management), health resilience, mass gatherings, public health, preparedness

\section{INTRODUCTION}

Mass gatherings (MG) are events that consist of a large number of people attending an activity or set of activities at a specific site or sites for a definite time period. The number of people can range from 1,000 to as many as 25,000 people. ${ }^{1}$ These MG can be planned or unplanned, recurrent or sporadic. These may be religious like the Hajj pilgrimage in Saudi Arabia, sports-related as in the Olympics and World Cup, entertainment-oriented like rock concerts, economic like world economic summits and political as in rallies and mass protests. ${ }^{2}$

Corresponding author: Ronald P. Law, MD, MPH

Regardless of the nature, MG are events that can Health Emergency Management Bureau Department of Health San Lazaro Compound, Tayuman, Sta. Cruz, Manila 1003 Philippines

Telephone: +639173446868

Email: rplaw@doh.gov.ph potentially challenge the capacities and strain the resources of countries and communities that are hosting them. Ultimately, MG can compromise the health system which in itself plays a crucial role in the preparedness and response to mass gathering incidents. ${ }^{3}$ Mass gatherings can lead to 
losses in lives of people through diseases and injuries, affect livelihood, and undermine health. ${ }^{4}$

Generally, MG can cause an increase in health risks. ${ }^{3,5}$ The following health risks have emerged from actual experiences with MG all over the world throughout history: gastrointestinal infections, respiratory infections, vectorborne disease, trauma and crush injuries, temperature-related conditions like heat exhaustion, hyperthermia, hypothermia, noncommunicable diseases, violence, and acts of terrorism leading to mass casualty incidents. ${ }^{5}$ These risks are related to challenges in crowd management, security, crush injuries, outdoor exposure, lack of food hygiene, inadequate waste management, poor sanitation, and violence. ${ }^{1}$

To address these, public health strategies should be put forward as regards advanced planning with a focus on building a multisectoral approach which puts health with other key sectors such as security, civil defense, and other response agencies.

In 2015, the Philippine Department of Health (DOH), being part of the government's multisectoral task force to provide medical support to mass gatherings by mobilizing medical teams on standby for health risks, was actively involved in the public health preparedness for the hosting of the following MG events: the Asia-Pacific Economic Cooperation (APEC) meeting, the Black Nazarene procession, and the Papal Visit. ${ }^{6}$

The study aimed to describe important health risks that may arise in mass gatherings and underscored the importance of public health preparedness strategies, policies, and measures that should be in place to reduce untoward health consequences.

\section{METHODS}

This was a descriptive study of the health risks that were observed throughout the conduct of the MG events. Health risks were health consequences that were attributed to the MG and were reflected as the reasons for consult. The source of data was the Surveillance in Post-Extreme Emergencies and Disasters (SPEED) database which records consultations for 21 syndromes of diseases whether communicable or noncommunicable. The criteria in reporting followed the prescribed SPEED reportable disease syndromes. The medical teams assigned to the different areas accomplished and submitted reports to the DOH Operations Center (Opcen) on a daily basis after each deployment day. The designated reporter of the teams accomplished the reporting forms while the team leader validated and cleared them for submission.
A total of 834 reports were collated and analyzed by Opcen staff who also extracted the data from the reports. The reports utilized a standard reporting form to facilitate extraction. The reports came only from medical teams deployed to the MG events and did not include data arising from possible consultations done in clinics and healthcare facilities outside of what the DOH had established. Figures were reported and presented for each MG event without respect to the total number of people who attended the MG. Further, consultation rates per disease syndrome were described as number of consultations for the disease syndrome over the total number of consultations for the MG. Quantitative data analysis was employed describing only the number of health risks observed in the MG events per consultation. Health risks were described both in terms of symptoms and diseases acknowledging the limitation of the SPEED as an early warning system. Upon submission to the Opcen, data validation, processing, and analysis were done by assigned emergency officers on duty with concurrence of Opcen supervisor. Data analysis was done with focus on health risks and implications for public health preparedness.

\section{RESULTS}

The APEC meetings ran for 11 months in 6 regions of the Philippines. Out of a total expected-14,700 people, 1,894 sought consultations. Medical problems reported included headache and hypertension as well as infectious conditions, especially gastrointestinal and respiratory. There were 6 referrals but no mortality was noted. (Table 1 ).

The Black Nazarene Traslacion happened for four days and was confined in Manila. Crowd estimate was put at around $14,853,680$ people. Of the total of 1,019 consultations, soft tissue injuries topped the list followed by medical problems such as dizziness, hypotension, hypertension, and difficulty of breathing. There were 17 referrals and 2 deaths, one due to acute coronary event and another to chronic liver disease.

The Papal Visit lasted for 5 days and took place in Manila and Tacloban, Leyte. It attracted around 3,500,000 people from all over the country and some parts of the world. A total of 2,384 consultations were reported. Hypothermia was the leading cause of consult, followed by respiratory infections, hypertension, and wounds. There were 2 mortalities - one was due to myocardial infarction and another due to injury or trauma.

For the APEC meetings, out of the total of 1,894 consultations, $27 \%$ was due to headache, $22 \%$ due to acute gastroenteritis, $19 \%$ to hypertension, $18 \%$ to upper

Table 1. Summary of health risks as to disease categories per consultation as reported by deployed medical teams in Philippine mass gatherings 2015, OPCEN data

\begin{tabular}{ccrrrr}
\hline Event & Date & Expected people & Consultations & \multicolumn{1}{c}{ Referrals } & Deaths \\
APEC & Dec 2014 to Nov 2015 & 14,700 & $1,894(12.88 \%)$ & $6(0.32 \%)$ & 0 \\
Black Nazarene Traslacion & Jan 7-10, 2015 & $14,853,680$ & $1,019(0.01 \%)$ & $17(1.67 \%)$ & $2(0.20 \%)$ \\
Papal visit & Jan 15-19, 2015 & $3,500,000$ & $2,384(0.07 \%)$ & $78(3.27 \%)$ & $2(0.08 \%)$ \\
\hline
\end{tabular}


Table 2. Reasons for consultation during APEC in 2015, OPCEN data $(n=1,894)$

Health risk

Headache

AGE

Hypertension

URTI

Others

AGE - Acute gastroenteritis

URTI - Upper respiratory tract infection

Table 3. Reasons for consultation during Black Nazarene Traslacion in 2015, OPCEN data $(n=1,019)$

\begin{tabular}{cc}
\hline Health risk & Number of consultations \\
\hline Soft tissue injury & $491(48 \%)$ \\
Dizziness/vertigo & $149(15 \%)$ \\
Hypotension & $91(9 \%)$ \\
Hypertension & $84(8 \%)$ \\
Difficulty of breathing & $61(6 \%)$ \\
Others & $143(14 \%)$ \\
\hline
\end{tabular}

Table 4. Reasons for consultation during Papal Visit in 2015, OPCEN data $(n=2,384)$

$\begin{array}{cc}\text { Health risk } & \text { Number of consultations } \\ \text { Hypothermia } & 537(23 \%) \\ \text { URTI } & 512(21 \%) \\ \text { Hypertension } & 449(19 \%) \\ \text { Wounds } & 375(16 \%) \\ \text { Fatigue } & 356(15 \%) \\ \text { Others } & 155(7 \%)\end{array}$

respiratory tract infection, and $13 \%$ was attributed to other syndromes (Table 2).

For the Black Nazarene procession, out of 1,019 consultations, $48 \%$ was due to soft tissue injury, and $15 \%$ due to dizziness/vertigo. These were followed by hypotension, hypertension and difficulty of breathing at $9 \%, 8 \%$, and $6 \%$, respectively; $14 \%$ was due to other syndromes (Table 3 ).

For the Papal Visit, out of 2,384 consultations, 23\% was due to hypothermia, $21 \%$ to upper respiratory tract infection and $19 \%, 16 \%$, and $15 \%$ to hypertension, wounds, and fatigue, respectively; $7 \%$ was due to other syndromes (Table 4).

\section{DISCUSSION}

The study of health risks of Philippine mass gatherings in 2015 revealed the following observations: First, the profile of common health risks evident in most MGs around the world surfaced as infectious causes, especially respiratory and gastrointestinal infections. For APEC meetings in particular, gastrointestinal infections were worth looking into. Respiratory infections in both APEC meetings and Papal Visit were significant. Trauma and injuries surfaced as equally important especially in the Black Nazarene procession wherein it topped the list of consultations but it deserved serious attention because one mortality in the Papal Visit was due to an injury, specifically head trauma.
Temperature-related conditions had also come to fore. For the Papal Visit, hypothermia was the leading cause of consultation. Hypertension, which could have been triggered by intense heat or cold, was also prominent in all of the 3 MGs. Headache which was the top cause of consultation during the APEC meetings could also be attributed to adverse temperature. For noncommunicable diseases, hypertension as a manifestation of cardiovascular diseases could be caused by stress and heat. Second, the findings highlight the importance of public health strategies in reducing the health risks of MGs. Food safety practices, water, sanitation and hygiene, proper advisories to people attending the events and surveillance systems are all deemed vital. Lastly, the analysis can arrive at the determination of factors to consider in planning for MGs. The profile of people attending the events by virtue of their nationality, age, gender, comorbidities, and other vulnerabilities should be studied to inform risk assessment for decision making. Environmental factors such as effects of weather disturbances, as evidenced by the impact of a typhoon that coincided with the Papal Visit, leading to hypothermia brought about by exposure of attendees to rains and cold wind should be considered. Hence, the site of the MG in terms of exposure to physical elements that can contribute to health risks as well as the location of the MG as far as the type of health system and existing health services in the area should also be taken into account. These will help reduce risk and facilitate preparedness of healthcare facilities during mass gatherings. In addition, the nature of MG (whether religious or economic for instance), level of management and coordination and type of resources available can be key determinants of the magnitude and manageability of incidents and health risks that might ensue.

\section{CONCLUSION}

Health risks that surfaced in selected mass gatherings in Philippines in 2015 validate the global experience and reflect the morbidity profile of the country which comprises both noncommunicable and infectious diseases. Therefore, traditional public health measures will be necessary. However, mass gathering preparedness entails strengthening of the health system to reduce potential of outbreaks and mass casualties and reduce burden of chronic diseases.

Just like any other emergency or disaster, MG events have to be approached using an all-hazards perspective so that hazard, exposure, and vulnerability parameters of people and their environment are addressed comprehensively and objectively guided by available evidence. ${ }^{4}$

Appropriate public health measures should be recommended to be able to handle effectively the public health risks of any MGs that the Philippines will host.

Inasmuch as MGs pose challenges to public health systems, these should be construed as opportunities for advancement as well. The Hajj in Saudi Arabia is responsible for improving the country's healthcare infrastructure and 
has also informed its global approach to public health. ${ }^{1}$ Lessons learned and good practices from these MGs should be viewed in the larger context of disaster risk reduction and management. The Philippines, having a significant experience in hosting and handling MGs should take advantage of these so that it can redound to improvements in its overall efforts to reduce disaster risks. Finally, this paper hopes to provide policy direction in relation to public health preparedness for mass gatherings.

\section{Statement of Authorship}

The author approved the final version submitted.

\section{Author Disclosure}

The author declared no conflict of interest.

\section{Funding Source}

None.

\section{REFERENCES}

1. Memish ZA, Stephens GM, Steffen R, Ahmed QA. Emergence of medicine for mass gatherings: lessons from the Hajj. Lancet Infect Dis. 2012;12(1):56-65.

2. Al-Tawfiq JA, Memish ZA. Mass gathering medicine: 2014 Hajj and Umra preparation as a leading example. Int J Infect Dis. 2014; 27:2631.

3. Memish ZA, Zumla A, Alhakeem RF, et al. Hajj: infectious disease surveillance and control. Lancet. 2014; 383(9934):2073-82.

4. Aitsi-Selmi A, Murray V, Heymann D, et al. Reducing risks to health and wellbeing at mass gatherings: the role of the Sendai Framework for disaster risk reduction. Int J Infect Dis. 2016; 47:101-4.

5. Joseph JK, Babu N, Dev KA, Pradeepkumar AP. Identification of potential health risks in mass gatherings: A study from Sabarimala pilgrimage, Kerala, India. International Journal of Disaster Risk Reduction. 2016; 17:95-9.

6. Valeza MCJ. Healthbeat Magazine, Department of Health. 2016;1:7-10. 